\section{Kikuchi-Fujimoto disease and breast implants: is there a relationship?}

Histiocytic necrotizing lymphadenitis, also known as Kikuchi-Fujimoto disease (KFD) is a subacute, necrotizing non-suppurative lymphadenitis that characteristically affects young adults, mostly women of Asian origin, while is more rarely observed in Western countries. ${ }^{1}$ KFD usually presents with isolated cervical lymphadenopathies, while constitutional symptoms are absent to mild. ${ }^{2}$ KFD has classically a favorable prognosis, although some cases with a more aggressive clinical course have been reported., The pathogenesis of KFD remains controversial. Hence, neither an infectious nor an autoimmune etiology have been confirmed so far; particular HLA class II alleles have been linked to KFD. ${ }^{6}$

Here, we report two cases of KFD occurring in patients with a history of bilateral silicone breast implants, highlighting the challenge that KFD poses to the differential diagnosis, particularly with T-cell lymphomas.

Case 1: A 40-year-old female with a history of bilateral breast augmentation with silicone implants for cosmetic reasons, developed an isolated left axillary lymphadenopathy five years after her breast surgery, which was diagnosed as ALK-negative anaplastic large cell lym- phoma (ALCL) at another institution. The patient underwent removal of her breast implants (which were negative for lymphoma at histologic examination) and was treated with six cycles of cyclophosphamide, hydroxydaunorubicin, vincristine and prednisone (CHOP). Six years later, she developed a second isolated, painless right laterocervical lymphadenopathy, tender and elastic in consistency. The case was suspicious for relapse and the patient underwent excisional biopsy at our institution. At this time, a review of the original diagnostic slides was also performed.

Case 2: The second patient is a 37 -year-old female, with an unremarkable past medical history who presented with an isolated, painless right cervical lymphadenopathy five years after a bilateral breast augmentation with silicone implants. An excisional biopsy of the lymph node was performed.

The lymph nodes of patient 1 and patient 2 were reviewed and a diagnosis of KFD was performed in both cases. In particular for patient 1, the original sample (i.e. originally diagnosed and treated as ALK-negative ALCL, was received for review) and the new sample (i.e. suspicious for relapse) revealed similar features of KFD. The patients received anti-inflammatory therapy as the only treatment and after a follow-up of 39 months and seven
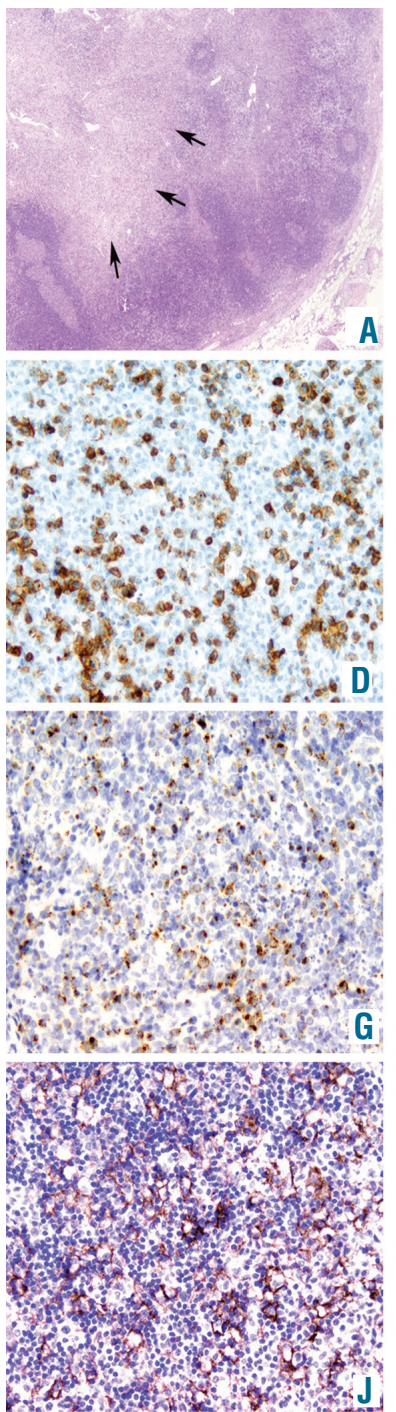
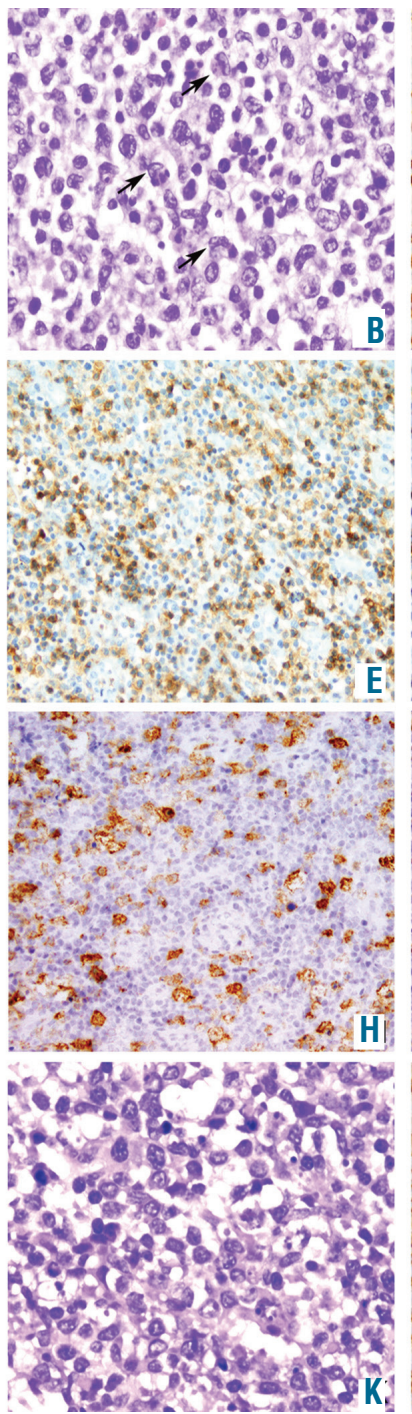

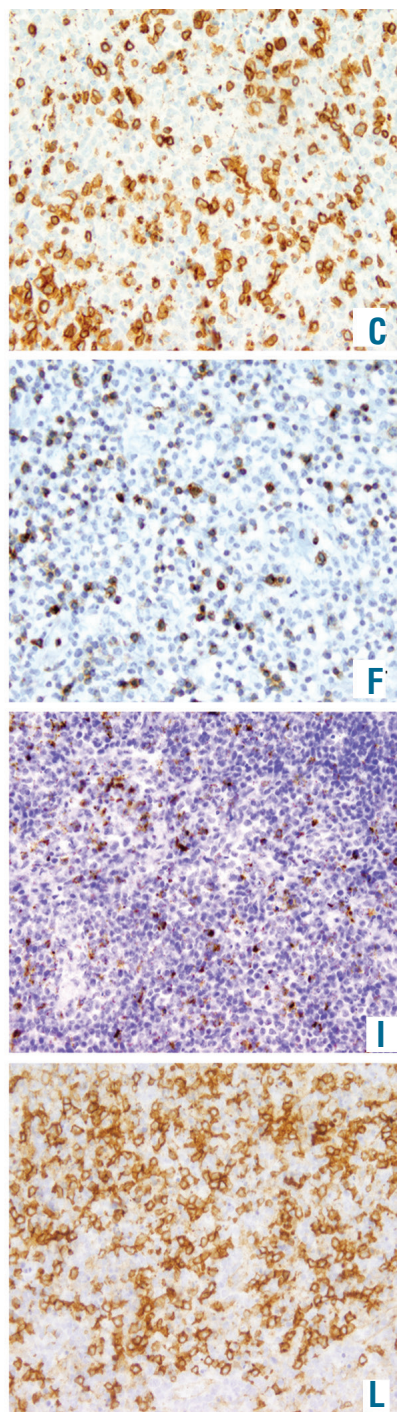

Figure 1. Histopathological and immunophenotypical features of the two Kikuchi-Fujimoto disease cases. A partial effacement of the architecture, with an expansion of the paracortex, pale areas of necrosis (black arrows) and residual lymphoid follicles are seen in both cases (A, H\&E). The necrotic areas contain abundant apoptotic debris, numerous histiocytes, some with reniform nuclei, so called "crescentic histiocytes" (black arrows) (B, H\&E). Both cases show an abundant population of CD3-positive T cells (C, CD3 $\mathrm{IHC}$ ), with a predominance of the CD8 (D, CD8 IHC) over CD4-positive T-cells (E, CD4 IHC). Case 1 shows a partial loss $(<50 \%)$ of CD5 (F, CD5 IHC). Abundant granzyme B-positive cytotoxic cells are identified in both cases (G, granzyme B IHC). Histiocytes express CD163 (H, CD163 IHC), with cytoplasmic co-expression of myeloperoxidase (I, myeloperoxidase $\mathrm{IHC}$ ) and PDL1 (J, PD-L1 IHC). Aggregates and sheets of plasmacytoid dendritic cells are observed in both cases (K, H\&E), which are positive for CD123 (L, CD123 IHC). Original magnifications: A: $1.25 x$; B, K: 40x; C-J, L: 20x. 
months respectively, no relapses have been documented. Microscopic examination of the excised lymph nodes revealed similar histologic features in the two cases. Lymph nodes showed a partial effacement of the architecture, with some residual follicles in the cortical zone. The paracortex was mottled in appearance, with pale areas of necrosis (Figure 1A), which showed extension to the perinodal tissue in case 1 . At high magnification, the necrotic areas contained mostly abundant apoptotic debris and were delineated by a mixed population of histiocytes, some with reniform nuclei (so called "crescentic histiocytes") and small to intermediate lymphocytes, a few with irregular nuclear contours (Figure 1B). Mitotic activity was brisk in isolated foci. In none of the two cases, empty vacuoles within the cytoplasm of histiocytes or a foreign body granulomatous reaction, suggesting a silicone lymphadenopathy, were seen.
Immunohistochemical studies showed abundant $\mathrm{CD}^{+}$ $\mathrm{T}$ lymphocytes with a predominance of the $\mathrm{CD}^{+}$over CD $4^{+}$cells. In case 1 , a partial loss $(<50 \%)$ of CD5 and CD7 was observed by comparing with CD3. The cytotoxic markers TIA-1, granzyme B and perforin were positive in numerous lymphocytes. Histiocytes were positive for CD4, CD68 (clone PG-M1), CD163, myeloperoxidase and PD-L1. CD123-positive plasmacytoid dendritic cells (pDCs) forming aggregates and sheets in the subcapsular and interfollicular areas were seen (Figure 1C-L). CD20 was positive in $\mathrm{B}$ lymphocytes within the residual follicles and in disperse immunoblasts in paracortical areas. Intriguingly, in case 1 numerous clusters of $\mathrm{CD}^{2} 0^{+}$atypical intermediate/large cells, some with prominent nucleoli and ample cytoplasm were seen in the subcapsular and interfollicular areas and similarly surrounding the necrotic areas. Double immunostaining for CD30 and

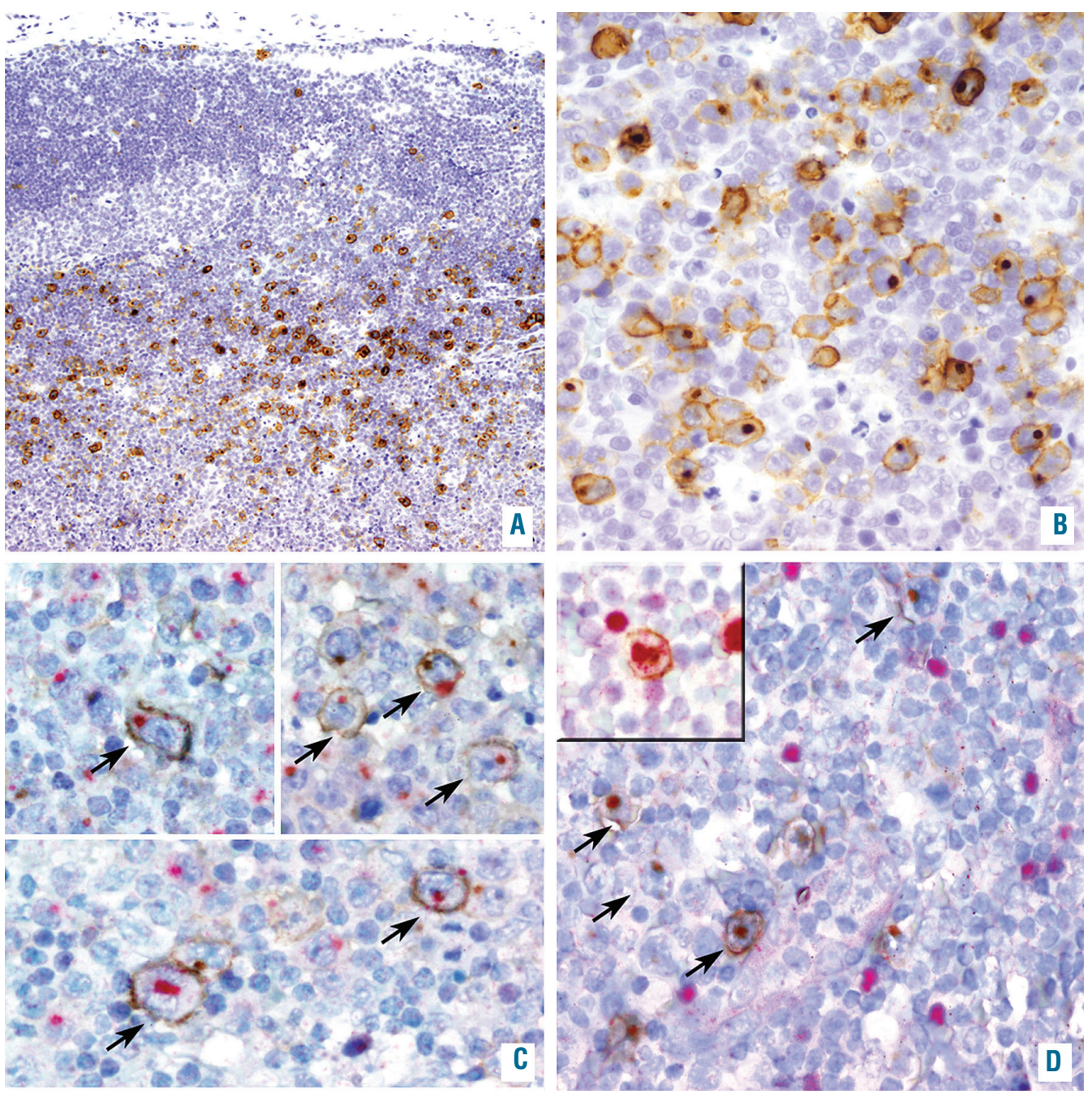

Figure 2. CD30-positive atypical cells in case 1. Case 1 showed numerous interfollicular clusters of CD30-positive atypical cells also surrounding the necrotic areas $(A, H \& E)$. CD $30^{+}$atypical intermediate/large cells show ample cytoplasm and some prominent nucleoli (B, H\&E). Composite image of double immunostainings with CD30 (brown) and granzyme B (red) demonstrated that most CD30 ${ }^{+}$atypical cells co-expressed granzyme B indicating that they corresponded to activated cytotoxic T cells (black arrows) (C, CD30 and granzyme B, double IHC), while they did not show co-expression of PAX5 (red) (black arrows) (D, CD30 and PAX5, double IHC). Tbet transcription factor (red) was positive in a proportion of CD30-positive cells (brown) (inset in D, CD30 and Tbet, double IHC). Original magnifications: A: 10x; B-D: 40x. 
CASE REPORTS

granzyme $\mathrm{B}$ demonstrated that most of the $\mathrm{CD} 30^{+}$atypical cells co-expressed granzyme $\mathrm{B}$ and the transcription factor Tret indicating that they corresponded to activated cytotoxic T cells, while they did not co-express of PAX5, GATA3 nor CD68 (Figure 2).

A molecular study of $B$ and $T$-cell receptor gamma (TAR $\gamma)$ gene rearrangements were negative for clonality in both cases. For case 1, TCR $\gamma$ gene rearrangement analysis was performed on both available samples (ie. original sample, diagnosed as ALCL and the new sample, suspicious for relapse). TCR beta (TCR $\beta$ ) gene rearrangement study was only performed in the second sample of case 1, in which a polyclonal pattern was observed (Figure 3).

Epstein-Barr virus (EBV) was negative by in situ hybridization with EBV-encoded RNA (EBER) probe. Similarly, the presence of HHV8 was not identified by LANA immunohistochemistry. The proliferation index
Ki67 was variable, reaching up to $80 \%$ in the areas surrounding the necrosis in both cases. Altogether the clinecal and histological findings were diagnostic of KFD in the so called "necrotic" phase.

We presented two cases of KFD that occurred in patients with a previous history of breast implantation. This finding has, first of all, an interesting pathogenic relevance, suggesting silicone implants may act as physicochemical agents triggering KFD.

Silicone implants elicit a chronic local immune response characterized by an infiltration of macrophages, dendritic cells, fibroblasts and activated $T$ cells mainly $\mathrm{CD}^{+}$(Th1/Th17-cells) with a decreased number of regtlatory $\mathrm{T}$ cells (Trees). ${ }^{7}$ To this regard, increased levels of cytokines IL-17, IL-6 and IL-2 have been detected in breast implant ALCL cell lines, supporting the role of chronic inflammation in the pathogenesis of "breast implant-associated ALCL".
A

First biopsy

TCR-Y

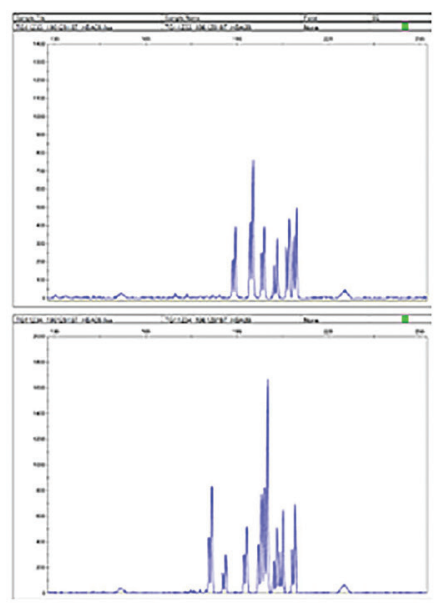

Figure 3. T-cell receptor rearrangement studies in case 1. Both the original sample, diagnosed as ALCL in another institution $(A)$ and the new sample, suspicious for relapse (B) were polyclonal for TCR $\gamma$ gene rearrangements. TCR $\beta$ gene rearrangement study was only performed in the second sample due to poor quaIity DNA (C).
B

Second biopsy

TCR-Y

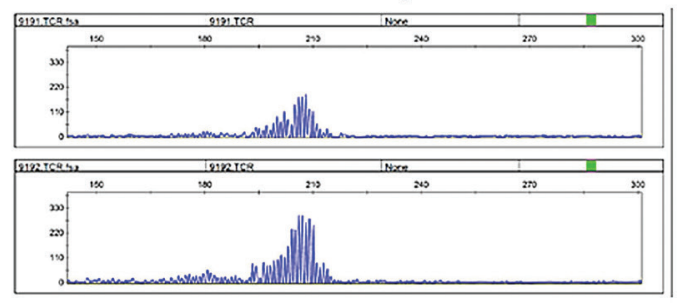

C

Second biopsy

TOR- $\beta$
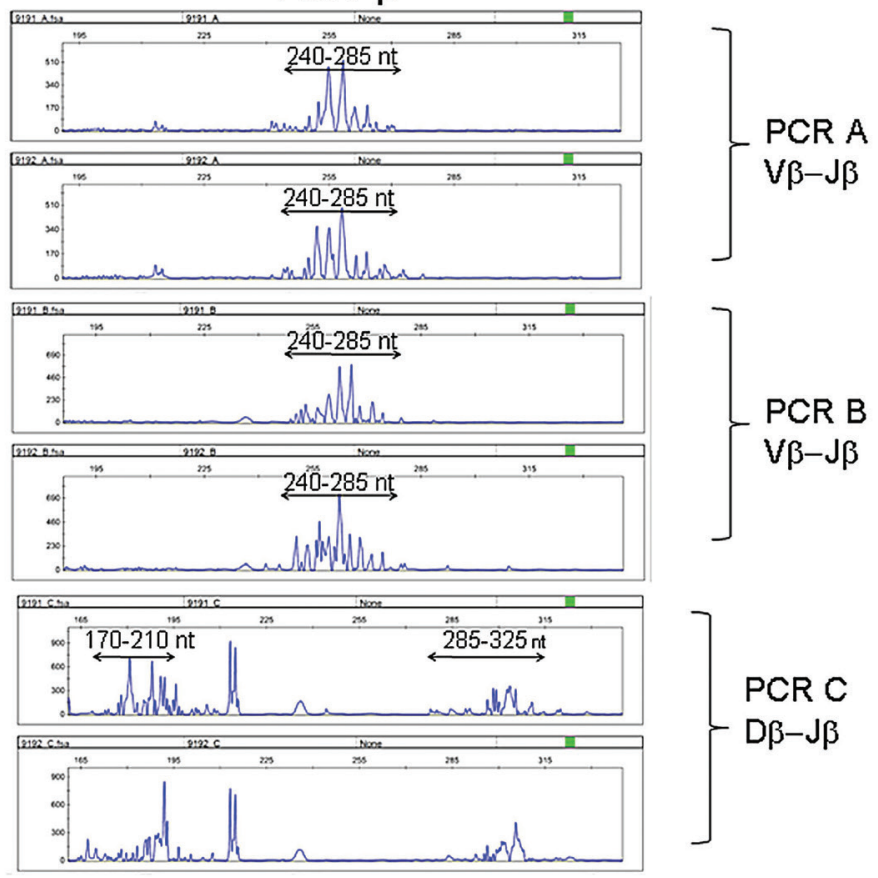

haematological 2019; 104:e583 
Additionally, the presence of a peculiar bacterial biofilm and a distinct microbiome in implants associated with ALCL have been speculated as a possible infectious contributing cause.

On the other hand, a certain dysregulation of the immune system is a well-known feature of KFD, with an excessive T-cell-mediated immune response, particularly involving $\mathrm{CD}^{+} \mathrm{T}$ lymphocytes. ${ }^{2} \mathrm{CD}^{+} \mathrm{T}$-cell function may be partially sustained by $\mathrm{pDCs}$, which also have a role in modulating or switching Tregs function and allowing Th1 and Th17 differentiation. These findings may explain in part the presence of abundant $\mathrm{pDCs}$ and $\mathrm{CD}^{+} \mathrm{T}$ cells in KFD. Increased serum levels of the proinflammatory cytokines IL-2, IL-6, IL-8, TNF- $\alpha$ and IFN- $\gamma$ have been detected in patients in the acute phase of KFD, and interestingly, the production of these cytokines may be stimulated by IL-17. ${ }^{10}$ Whether IL-17 and Th17 T cells play a role in KFD needs further research.

Even if infectious agents are the most speculated, some physicochemical agents have been reported, anecdotally, as possible causative agents of KFD. Charalabopoulos and coworkers reported one case of KFD occurring in a 64-year-old Caucasian female six weeks after a pacemaker implantation. ${ }^{11}$ Similarly, Sever et al. described a simultaneous silicone lymphadenopathy and KFD affecting an axillary lymph node as a consequence of a leaking silicone breast implant. ${ }^{12}$ However, in this case a leaking silicone breast implant was observed whilst this was not the case in our two patients. In our cases, KFD occurred in patients with a history of silicone breast implants. Hence, a pro-inflammatory milieu is a common feature in both entities, but whether implants may elicit a local deregulated immune response leading to the development of KFD in exceptional cases deserves further research.

At the same time, our cases underlie the issue of a differential diagnosis of KFD. Case 1 had an initial diagnosis of ALK-negative ALCL affecting an axillary lymph node and treated with CHOP. Six year later, the patient developed a laterocervical lymphadenopathy, clinically suspicious for relapse. A review of the first diagnostic sample was consistent, instead, with KFD. KFD mimicking malignant lymphoma is a well- known, but never overrated pitfall. ${ }^{13}$ This is particularly true for KFD in its early, "proliferative" phase, characterized by a florid proliferation of large lymphoid cells, with prominent nucleoli which can raise the differential diagnosis of large cell lymphoma. ${ }^{14}$ Moreover, $\mathrm{T}$ lymphocytes in KFD may show aberrant T-cell antigen loss, ${ }^{15}$ another possible misleading feature. This differential diagnosis is of particular importance, giving the striking difference in clinical management. Relevant features that should suggest KFD are a patchy rather than diffuse nodal involvement, the presence of a "clean" necrosis with no neutrophils and the characteristic coexpression of myeloperoxidase, the absence of anaplastic, "hallmark" cells and the collections of CD123 positive pDCs. Identification of CD30-positive cells should not be misinterpreted as suggestive of malignancy as this is a well-known finding in KFD. In our cases, we demonstrated that these cells correspond to activated, cytotoxic $\mathrm{T}$ cells. Absence of clonality by a $T C R$ study would point towards a reactive condition. However, in KFD a clonal TCR rearrangement can be detected, therefore whatever the result, the diagnosis should not change at least in an expert environment.

In conclusion, the pathogenesis of KFD is still unknown. Here, we reported two cases of KFD occurring in the setting of silicone breast implants. Whether silicone breast implants may act as physicochemical agents triggering KFD deserves further investigation as the number of cases reported is too limited and anecdotal. A certain degree of morphologic overlapping between KFD and malignant lymphomas does exist; hence a careful histological examination and an integration with clinical findings are pivotal to achieve a correct final diagnosis.

Valentina Sangiorgio, ${ }^{1}$ Luis Veloza, ${ }^{2}$ Karen Galvis, ${ }^{3}$ Mónica López, ${ }^{2}$ Gerard Frigola, ${ }^{2}$ Elias Campo and Olga Balaguéz

${ }^{1}$ Department of Cellular Pathology, Barts and The London NHS Trust, London, UK; ${ }^{2}$ Haematopathology Unit, Pathology Department, Hospital Clinic of Barcelona, University of Barcelona, Villarroel, Barcelona, Spain and ${ }^{3}$ Department of Pathology and Laboratory Medicine, Fundación Santa Fe de Bogotá University Hospital, Bogotá, Colombia

Correspondence: VALENTINA SANGIORGIO

valentina.sangiorgio@nhs.net

doi:10.3324/haematol.2019.229831

Information on authorship, contributions, and financial \& other disclosures was provided by the authors and is available with the online version of this article at www. haematologica.org.

\section{References}

1. Dorfman RF. Histiocytic necrotizing lymphadenitis of Kikuchi and Fuijmoto. Arch Pathol Lab Med. 1987;111(11):1026-1029.

2. Bosch X, Guilabert A, Miquel R, Campo E. Enigmatic KikuchiFujimoto disease: a comprehensive review. Am J Clin Pathol. 2004; 122(1):141-152.

3. Jain J, Banait S, Tiewsoh I, Choudhari M. Kikuchi's disease (histiocytic necrotizing lymphadenitis): A rare presentation with acute kidney injury, peripheral neuropathy, and aseptic meningitis with cutaneous involvement. Indian J Pathol Microbiol. 2018;61(1):113-115

4. Sato Y, Kuno H, Oizumi K. Histiocytic necrotizing lymphadenitis (Kikuchi's disease) with aseptic meningitis. J Neurol Sci. 1999; 163(2):187-191.

5. Chong Y, Kang CS. Causative agents of Kikuchi-Fujimoto disease (histiocytic necrotizing lymphadenitis): a meta-analysis. Int J Pediatr Otorhinolaryngol. 2014;78(11):1890-1897.

6. Tanaka T, Ohmori M, Yasunaga S, et al. DNA typing of HLA class II genes (HLA-DR, -DQ and -DP) in Japanese patients with histiocytic necrotizing lymphadenitis (Kikuchi's disease). Tissue Antigens. 1999; 54(3):246-253

7. Wolfram D, Rabensteiner E, Grundtman C, et al. T regulatory cells and TH17 cells in peri-silicone implant capsular fibrosis. Plast Reconstr Surg. 2012;129(2):327e-337e.

8. Brody GS, Deapen D, Taylor CR, et al. Anaplastic large cell lymphoma occurring in women with breast implants: analysis of 173 cases. Plast Reconstr Surg 2015;135(3):695-705.

9. Hu H, Johani K, Almatroudi A, et al. Bacterial biofilm infection detected in breast implant-associated anaplastic large-cell lymphoma. Plast Reconstr Surg. 2016;137(6):1659-1669.

10. Szturz P, Adam Z, Chovancová J, et al. Cytokine analysis in a patient with relapsing Kikuchi-Fujimoto disease. Leuk Lymphoma. 2012; 53(4):743-745

11. Charalabopoulos K, Charalabopoulos A, Binolis J, Papalimneou V, Ioachim E. Is implant pacemaker a physicochemical cause triggering Kikuchi-Fujimoto disease? In Vivo. 2002;16(1):73-76.

12. Sever CE, Leith CP, Appenzeller J, Foucar K. Kikuchi's histiocytic necrotizing lymphadenitis associated with ruptured silicone breast implant. Arch Pathol Lab Med. 1996;120(4):380-385

13. Chamulak GA, Brynes RK, Nathwani BN. Kikuchi-Fujimoto disease mimicking malignant lymphoma. Am J Surg Pathol. 1990;14(6):514523.

14. Kuo TT. Kikuchi's disease (histiocytic necrotizing lymphadenitis). A clinicopathologic study of 79 cases with an analysis of histologic subtypes, immunohistology, and DNA ploidy. Am J Surg Pathol. 1995; 19(7):798-809.

15. Wei X-J, Zhou X-G, Xie J-L, Zheng X-D, Zheng Y-Y. Aberrant phenotypes in Kikuchi's disease. Int J Clin Exp Pathol. 2014;7(9):5557 5563. 